\title{
Efficiency of two different transfection reagents for use with human NTERA2 cells
}

\author{
M.G. Aluigi, ${ }^{1}$ S. Hofreiter, ${ }^{2}$ C. Falugi, ${ }^{1}$ M. Pestarino, ${ }^{1}$ S. Candiani ${ }^{1}$ \\ ${ }^{1}$ Department of Biology, University of Genova, Genova, Italy; ${ }^{2}$ Biontex Laboratories GmbH, \\ Martinsried/Planegg, Germany
}

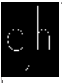

C2007 European Journal of Histochemistry

The teratocarcinoma cell line NTERA2 is recently used in a wide range of researches (from developmental biology to toxicology, for their ability to be induced to neural differentiation.

In order to study the genetic potential of these cells, it is needed to use methods for gene silencing and/or mRNA interference, allowing cell viability and further differentiation. To check these features, we simultaneously tested the transfection efficiency of NTERA2, A549 and HeLa cells with Metafectene PRO (Biontex, Germany) and another optimal transfection reagent currently used in our Laboratory, using as a reporter gene the DsRed2 vector (Clontech, Mountain View, CA). Under our culture conditions for NTERA2 and HeLa cells, Metafectene PRO transfection method was found to possess high throughput performance, that allows low concentration rate and low exposure time to excitation light source, thus reducing both toxicity and phototoxicity.

Key words: transfection, efficiency, toxicity, phototoxicity.

Correspondence: Mario Pestarino,

Department of Biology, University of Genova,

Viale Benedetto XV, 5. I-16132 Genova, Italy

Tel. +39.010.3538043.

Fax. +39.010 .3533062$

E-mail: pesta@unige.it

Paper accepted on November 16, 2007

European Journal of Histochemistry

2007; vol. 51 issue 4 (October-December):301-304
T he teratocarcinoma cell line NTERA2 is a human embryonic cell line, used in a wide range of researches (from developmental biology to toxicology), for their ability to be induced to pluripotent differentiation.

In order to study the potential of these cells, gene modification is needed to check the effects of silencing or expressing genes, without compromising viability or further differentiation.

Cell transfection is used in biomedical and biological research to study the role of individual gene products in vitro or in vivo. Viral and non-viral gene transfer systems are available from several suppliers, and several cell lines and primary cells can efficiently be transfected (Zhang et al., 2001; Banerjee et al., 2001).

For NTERA2 cells, the method mainly used to get transfection is up-to-date infection by viral vectors.

In this work, we examined the efficacy of a novel transfect ion reagent Metafectene (Biontex, Germany) using DsRed2 vector (Clontech, Mountain View, CA) in human NTERA2 cells as compared to other cell lines, such as HeLa cells and A549 cells.

Comparison among the different cell lines was carried out because, while HeLa and A549 cells are rather stable, cultured NTERA2 cells are difficult to transfect, being very sensitive to culture conditions. Actually, the pluripotent NTERA2 cell lines are used to check differentiation, and a high degree of survival is needed.

We established the concentrations of Metafectene causing efficiency of transfection without signs of cell degeneration that are frequently observed when using calcium phosphate or electroporation.

The efficiency/toxicity balance was compared with a non-liposomal multicomponent transfection medium, previously routinely used in our Laboratory.

This work is carried out in the context of a research aimed at using NTERA2 cells as a material for the study of neural differentiation. 


\section{Materials and Methods}

\section{Cell lines}

The human cervical cancer cell line HeLa and the human teratocarcinoma cell line NTERA2-D1 were obtained from the American Type Culture Collection (ATCC) (Rockville, MD) and maintained in an atmosphere of $5 \% \mathrm{CO}_{2}$, at $37^{\circ} \mathrm{C}$ in Dulbecco's modified Eagle's MEM (DMEM) supplemented with $10 \%$ FCS (Celbio, Italy), 1 mM L-glutamine and antibiotics (100 units/mL penicillin and 100 $\mu \mathrm{g} / \mathrm{mL}$ streptomycin).

The human lung carcinoma cell line A549 were obtained from the ATCC (Rockville, MD) and maintained in an atmosphere of $5 \% \mathrm{CO}_{2}$, at $37^{\circ} \mathrm{C}$ in RPMI-1640 supplemented with 10\% FCS (Celbio, Italy), $1 \mathrm{mM}$ L-glutamine and antibiotics (penicillin and streptomycin).

\section{Transfection protocol}

For transiently transfection with a red fluorescent protein, HeLa $\left(1 \times 10^{5}\right.$ cells/well), NTERA2 $\left(1 \times 10^{5}\right.$ cells/well) and $A 549\left(4 \times 10^{4}\right.$ cells/well) cell lines were seeded on poly-L-lysine coated slides (Sigma Chem Co., Saint Louis, M0) in $1 \mathrm{~mL}$ of medium in 12-well culture plates, resulting in about $80-90 \%$ confluency within 24 hours. Cells were transfected one day later with DsRed2 vector (Clontech, Mountain View, CA) using Metafectene PRO (Biontex, Germany) and a transfection reagent routinely (TRR) used in our laboratory according to the manufacturer's instructions.

Cells were pre-washed with PBS and then covered with $1 \mathrm{~mL}$ of serum-free medium. Metafectene PRO was complexed with the expression construct. The metafectene solution and construct (DNA) solution was prepared separately mixing Metafectene PRO/DNA with $50 \mu \mathrm{L}$ serum free medium (ratio Metafectene PRO/DNA 2:1, 4:1, $6: 1$ ), and after that the two solution were mixed. The mixture was incubated for $15 \mathrm{~min}$ at room temperature and then added in a volume of $0.1 \mathrm{~mL}$ per well. The cells were incubated in serum-free DMEM or RPMI 1640 and after $24 \mathrm{~h}$ of transfection were fixed with $4 \%$ paraformaldehyde $(\mathrm{pH} 7.4)$ for 10 min, and then stained with Hoechst 33258 (5 $\mathrm{mg} / \mathrm{L}$ ) for $10 \mathrm{~min}$ at room temperature and washed twice in distilled water. The coverslips were removed and mounted on glass microscope slides with SuperMount $^{\circledast}$ (BioGenex, San Ramon, CA) to achieve optimal fluorescence.
RFP-cells (red fluorescent positive cells) were analyzed using an Olympus IX71 microscope (Olympus Italia s.r.l., Italy). Images were acquired at the excitation wave length of $540 \mathrm{~nm}$ and processed using the analySIS software package (Soft Imaging System GmbH, Germany). Cell morphology was evaluated by inverted phase contrast microscopy at 10-20x magnification.

\section{Cytotoxicity assay}

To determine cell lines viability, a MTT assay [3(4,5-dimethylthiazol-2-yl)-2,5-diphenyl tetrazolium bromide] (Mosmann. 1983) was used. Briefly, HeLa $\left(1 \times 10^{5}\right.$ cells/well), NTERA2 $\left(1 \times 10^{5}\right.$ cells/well) and $A 549\left(4 \times 10^{4}\right.$ cells/well) cell lines were seeded into 12 wells plates and prior to the 3hour MTT $(5 \mathrm{mg} / \mathrm{mL}$ in HBSS/HEPES [Hank's Balanced Salt Solution/HEPES] [Sigma Chem. Co., MO]) treatment, the cells were exposed for 24 hours to the Metafectene PRO and transfection reagent routinely used in our laboratory formulations at the same end concentrations used for the transfection experiments.

The MTT assay was performed according to the manufacturer's instructions. The remaining viable cells with MTT dye uptake were determined by measuring the optical density at $520 \mathrm{~nm}$ in a spectrophotometer (6405 Ultra Violet/Visible, Barloworld Scientific Ltd, United Kingdom).

\section{Results}

\section{Transfection efficiency}

In HeLa Cells, the transfection efficiency was very high: at concentration 2:1 the ratio of transfected cells vs non transfected cells was approximately 30-35\% both with TRR used and Metafectene PRO (Figure 1): DsRed2 vector displays a consistent and homogeneous distribution when transfected in HeLa cells.

In contrast, transfection of lung carcinoma cell line A549 provided no mentionable RFP-positive cells, either with Metafectene PRO or with the current transfection method (not shown).

In NTERA2 cells (Figure 2), the transfection efficiency of Metafectene PRO was similar to that obtained in HeLa cells. We measured the transfection efficiency by comparing the exposure times needed to acquire the images by use of the analySIS software: Metafectene PRO transfected cells were acquired with an exposure time lower 
(500 ms at 20X magnification) than of TRR transfected cells ( $1 \mathrm{~s}$ at $20 \mathrm{X}$ magnification) (Figure $3 \mathrm{~A}$ ). This also allows to decrease possible phototoxicity when experiments on living cells are needed.

\section{Cytotoxicity}

In HeLa cells, Metafectene PRO and TRR revealed no toxicity in either concentration used, as determined microscopically (Figure $1 \mathrm{~B}, \mathrm{D}, \mathrm{F}, \mathrm{H}$ ) and by MTT assay (Figure 3B).
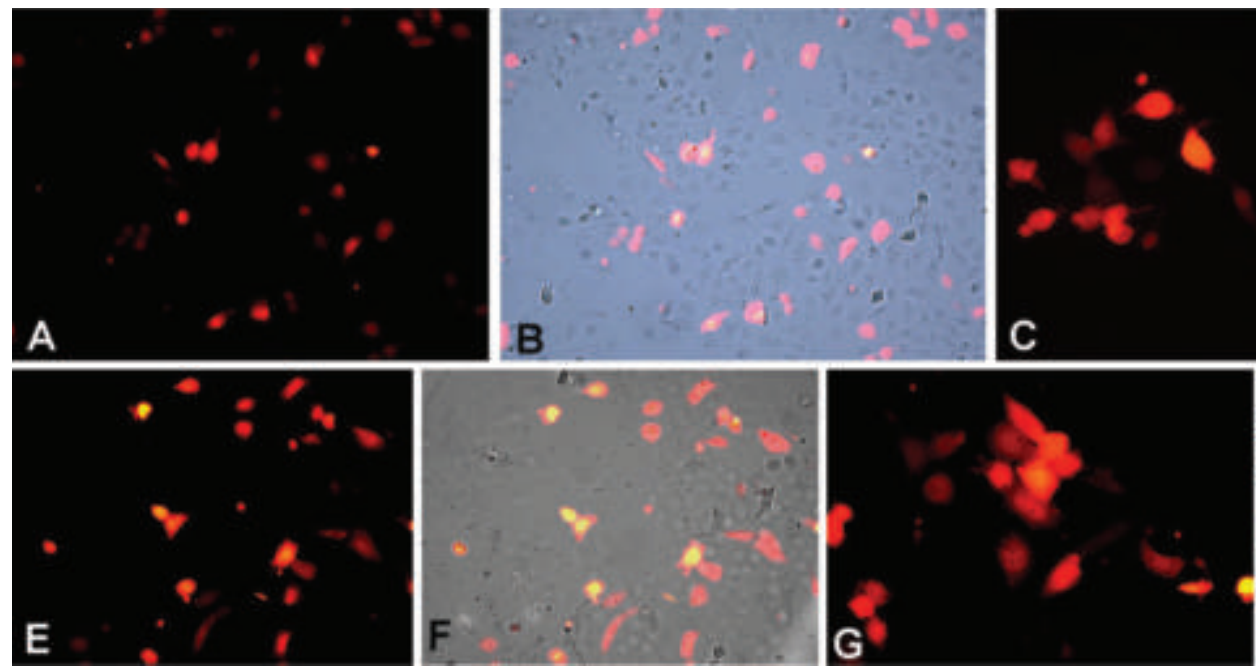

O
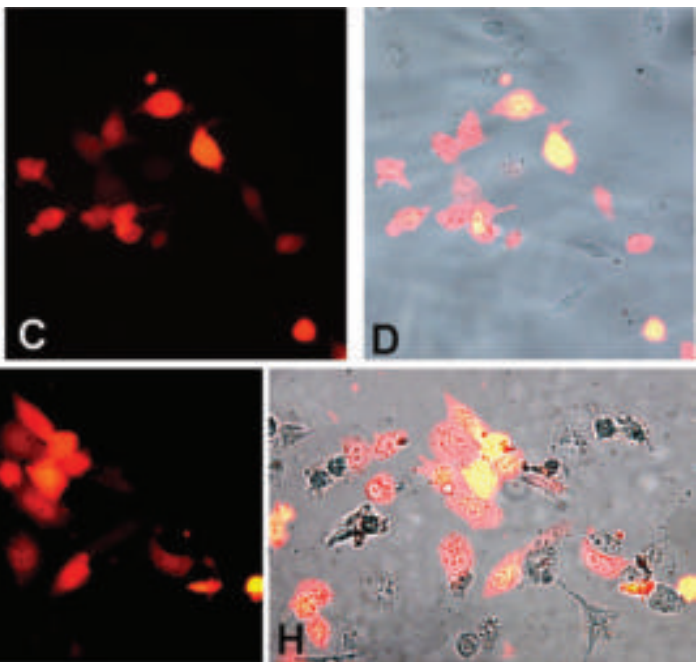

Figure 1. A-D, Hela transfected cells with TRR. E-H, Hela transfected cells with Metafectene PRO. A,C,E,G Red fluorescent Hela cells. B, D, F, H Morphological.
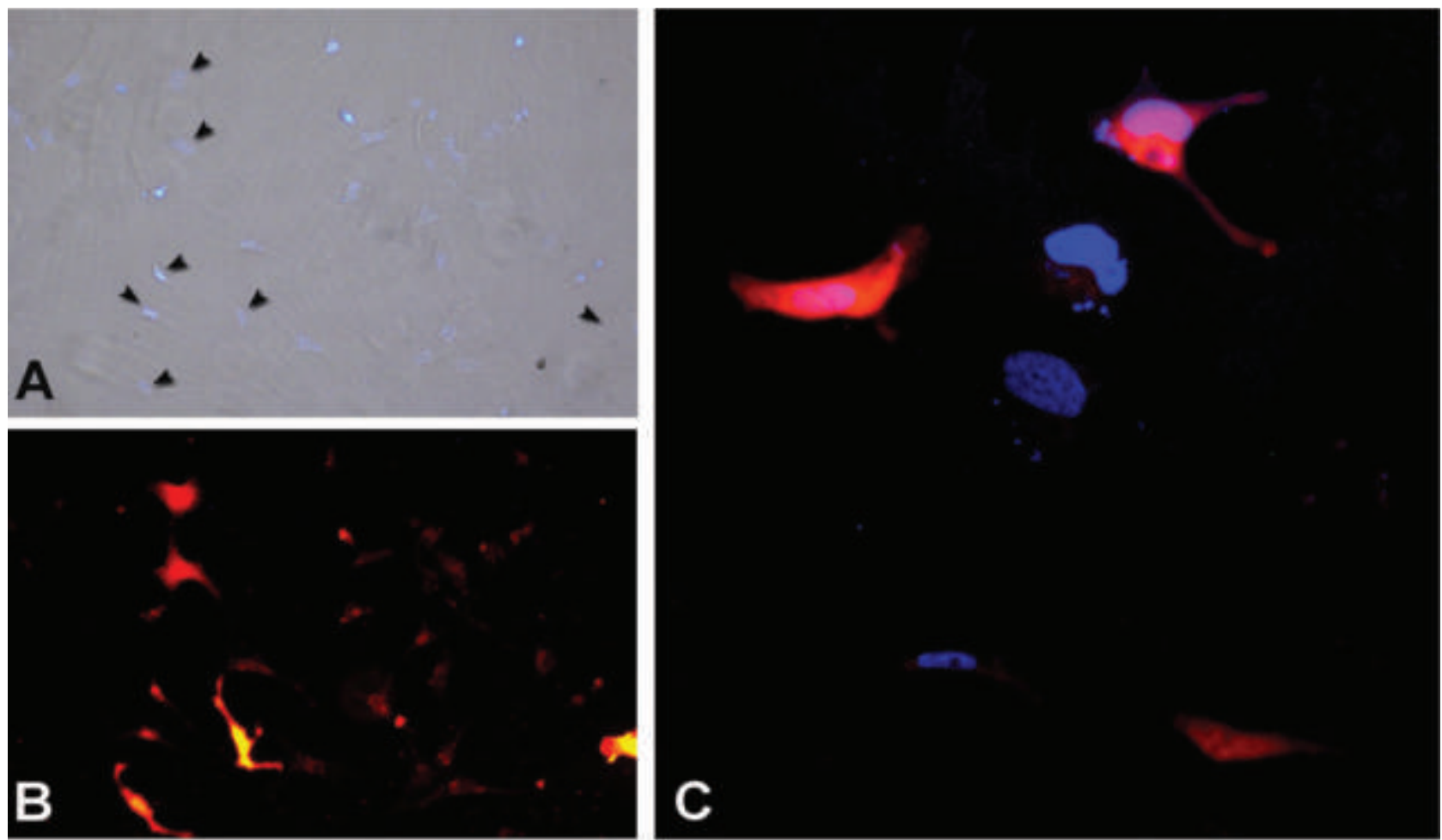

Figure 2. NTERA2 transfected cells with Metafectene PRO. A, Morphological images of NTERA2 cells at 10X magnification. B, Cells indicated by arrowheads in A positive for DsRed2. C, A particular of NTERA2 cells RFP-positive and where the nuclei are counterstained in blue with Hoechst 33258 (20X magnification). 


\section{A fluorescence intensity measured by exposure time}

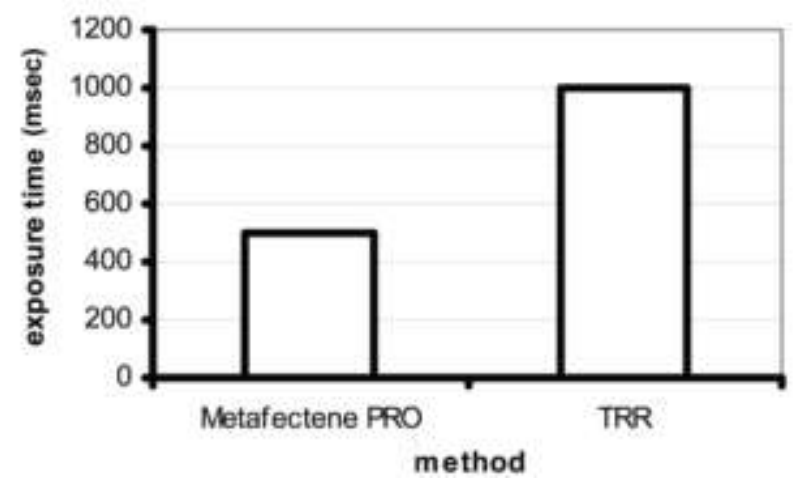

\section{B MTT: surviving cells after treatment}

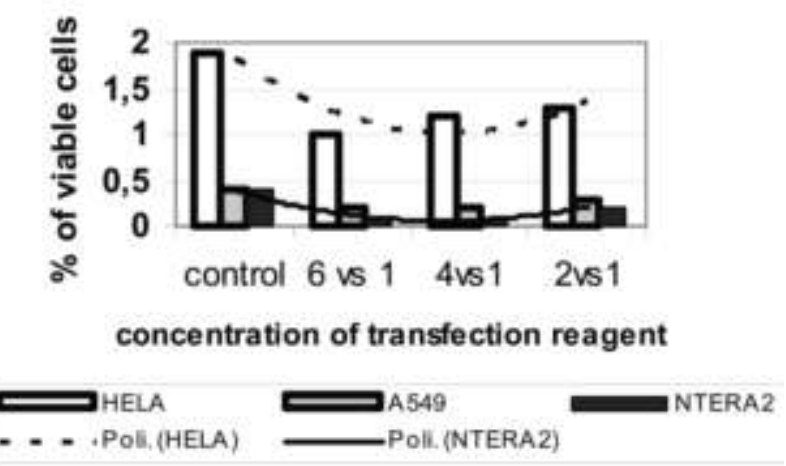

Figure 3. Difference between Metafectene PRO and TRR transfection efficiency. Y axis: fluorescence intensity measured by the camera exposure time of the AnalySis software. Standard deviation=0. B: MTT showing surviving cell rates at the different concentrations of Metafectene PRO vs plasmid. Control= non transfected cells: the polynomial trend lines show that the effect of transfection is the same for both HeLa and NTERA2 cells, the differences being due to the native different proliferation rates of the two cell lines.

NTERA2 seem to be more susceptible to the toxic effect of Metafectene PRO (4:1 and 6:1) as compared to HeLa cells. Nevertheless, the fact that Metafectene PRO allows a good transfection also at lower concentrations (2:1) allows to obtain a good transfection with low toxicity.

\section{Discussion}

An optimized protocol for mammalian adherent cells transfection was developed, based on polycationic liposomal transfection reagent, Metafectene PRO. Using the DsRed2 vector and Metafectene PRO Transfection Reagent resulted in efficient transfection of the teratocarcinoma cell line NTERA2 and cervical cancer cell line HeLA with the optimum around Metafectene PRO/DNA ratio of 2:1 after 24 hour incubation. As for all the transfection methods, there is a clear inverse relationship between transfection efficiency and toxicity; anyway, thank to the high transfection performance, Metafectene PRO seems a very good reagent to detect expressed fluorescent protein with low concentration and low exposure time. The transfected NTERA2 cells retained their viability and proliferation ability at the same rate as the
HeLa. Thus, both chemical and phototoxicity may be reduced and this allows a very good throughput of the transfection. The ability NTERA2 cells, as a good surrogate of stem cells, to differentiate into specific cell types holds a potential for studying the basis of stem cell therapeutic use in cell and gene therapy. Realization of this potential depends on efficient and optimized protocols for genetic manipulation of cultured cells.

\section{Acknowledgments}

We warmly thank Dr. Patrizia Russo (INRC Genova, Italy) for the gift of A549 cell line and Dr. Patrizio Castagnola (INRC Genova, Italy) for the gift of the DsRed2 vector.

\section{References}

Banerjee R, Mahidhar YV, Chaudhuri A, Gopal V, Rao N M. Design, synthesis and transfection biology of novel cationic glycolipids for use in liposomal gene delivery. J Med Chem 2001; 44:4176-85.

Mosmann T. Rapid colorimetric assay for cellular growth and survival: application to proliferation and cytotoxicity assays. J Immunol Methods 1983; 65:55-63.

Zhang G, Budker VG, Ludtke JJ, Wolff JA. Naked DNA gene transfer in mammalian cells. Methods Mol Bio 2004; 245:251-64. 